\title{
Potential Roles of Protease Inhibitors in Cancer Progression
}

\author{
Peng Yang ${ }^{1}$, Zhuo-Yu Li ${ }^{1,2}$, Han-Qing $\mathrm{Li}^{3 *}$
}

\begin{abstract}
Proteases are important molecules that are involved in many key physiological processes. Protease signaling pathways are strictly controlled, and disorders in protease activity can result in pathological changes such as cardiovascular and inflammatory diseases, cancer and neurological disorders. Many proteases have been associated with increasing tumor metastasis in various human cancers, suggesting important functional roles in the metastatic process because of their ability to degrade the extracellular matrix barrier. Proteases are also capable of cleaving non-extracellular matrix molecules. Inhibitors of proteases to some extent can reduce invasion and metastasis of cancer cells, and slow down cancer progression. In this review, we focus on the role of a few proteases and their inhibitors in tumors as a basis for cancer prognostication and therapy.
\end{abstract}

Keywords: Protease - protease inhibitor - tumor metastasis - targeted cancer therapy.

Asian Pac J Cancer Prev, 16 (18), 8047-8052

\section{Introduction}

One of the basic characteristics of tumor cells is their capability to invade into normal tissue (Hanahan and Weinberg, 2011). After shedding themselves off from the primary tumor, malignant cells invade into the extracellular matrix (ECM) and basement membrane (BM). Moreover, these cells adhere to some molecules of intercellular substance, activate cells to synthesize various kinds of degrading enzymes to assist the tumor cells enter into the blood vessel through the ECM (Lu et al., 2012; van Horssen et al., 2013). New blood vessels are essential for the growth of primary tumor and metastasis, where they continue to proliferate and form a metastatic colony. It is conceivable that proteolytic degradation of ECM plays a crucial role in the tumor development (Alitalo and Detmar, 2012). ECM is a dense network composed of laminin, fibronectin and other glycoproteins, collagens, and proteoglycans (Lu et al., 2012). The invasion and metastasis process of tumor cells is through a lytic machinery that composed of different proteolytic enzymes, named as the proteases. They are subdivided into five categories: matrix metalloproteases (MMPs), cystine proteases, serine proteases, aspartic proteases and threonine proteases (Sanman and Bogyo, 2014). The main classes of proteases that contribute to the lytic processes around tumors are MMPs, cathepsins (cystine proteases), and plasminogen activators (serine proteases) (Table 1).

Each class of proteases has natural inhibitors which modulate their activity, such as, tissue inhibitors of metalloproteinase (TIMPs), the cystatins, which inhibit cathepsins, and the plasminogen activator inhibitors (Rakashanda et al., 2013). The proteolytic enzymes are first secreted as inactive proenzymes, and then become activated by proteolytic cleavage. Other factors have bidirectional roles in proteolytic enzyme cascade. For example, Basic fibroblast growth factor, released from the protein hydrolysis mediated by plasmin, can induce the expression of protease and other related factors in tumor cells and endothelial cells, forming loops in the proteolytic cascade (Hart et al., 2011).

The development of malignant tumors is characterized by at least five major processes: proliferation, apoptosis, angiogenesis, invasion and migration of cells (Figure 1). In healthy tissue, there is an equilibrium between cell division and programmed cell death (apoptosis). In tumors, this equilibrium is disturbed by increased cell division, decreased apoptosis or both (Gupta et al., 2010). When atumor reaches a certain size, it becomes dependent on the growth of new blood vessels, angiogenesis for a constant supply of oxygen and nutrients and removal of waste products. Various processes are involved in invasion and migration, including loss of cell-cell and cell-matrix adhesion and degradation of extracellular matrix (ECM) components (Revach and Geiger, 2014). When the expression of cell-cell and cell-matrix adhesion molecules is reduced or missing, cells lose contact with their microenvironment and are predisposed to invade and migrate into surrounding tissue. Malignant cells show increased proteolytic activity, which helps them digest the ECM. This digestion is required for cancer cells to invade and migrate through the basal lamina,

${ }^{1}$ Institute of Biotechnology, Key Laboratory of Chemical Biology and Molecular Engineering of National Ministry of Education, Shanxi University, ${ }^{3}$ College of Life Science, Shanxi University, Taiyuan, ${ }^{2}$ College of Life Science, Zhejiang Chinese Medical University, Hangzhou,China*For correspondence:lzy@sxu.edu.cn 
which is the hallmark of malignancy (Yadav et al., 2014). Invasion and migration of cancer cells may lead to the development of metastases at distant sites (Zongwei and Hong, 2013.02.002). Metastases are the major cause of death in cancer patients. Therefore, reduction of metastatic progression is the greatest challenge in the development of effective anticancer therapies.

\section{Expression and Chemotherapy of MMPs}

MMPs are a group of zinc-dependent endopeptidases that involve in the degradation of the ECM components and play a key role in ECM homeostasis (Gialeli et al., 2011). According to its substrate specificity, over 25 human MMPs are divided into five categories: collagenases, gelatinases, stromelysins, elastases and membrane type MMPs (Behm et al., 2012). Recent studies have found that MMPs have a wide range of functions that include regulating cell growth and apoptosis, triggering the release of growth factors, altering cell motility, affecting immune responses and regulating the activity of cytokines and chemokines (Yang et al., 2014; Shay et al., 2015). Furthermore, MMPs have also been correlated with cancer dissemination and tumor angiogenesis (Shuman et al., 2012).

The levels of MMPs are tightly regulated at many stages including transcription, epigenetic alterations, activation from precursor zymogens (post-translational) as well as inhibition by TIMPs (Yan and Boyd, 2007). Several transcription factors, including AP-1, PEA3, NF$x \mathrm{~B}, \mathrm{STAT} 3, \beta$-catenin and Sp-1, have been identified to directly upregulate MMPs gene transcription (Nissinen and Kahari, 2014). Various cytokines or growth factors, including EGF, TNF- $\alpha$ and TNF- $\beta$, may act on these transcription factors to indirectly promoting MMPs gene transcription (Gearing et al., 1994). MMPs can be targeted by a growing number of miRNAs at posttranscriptional

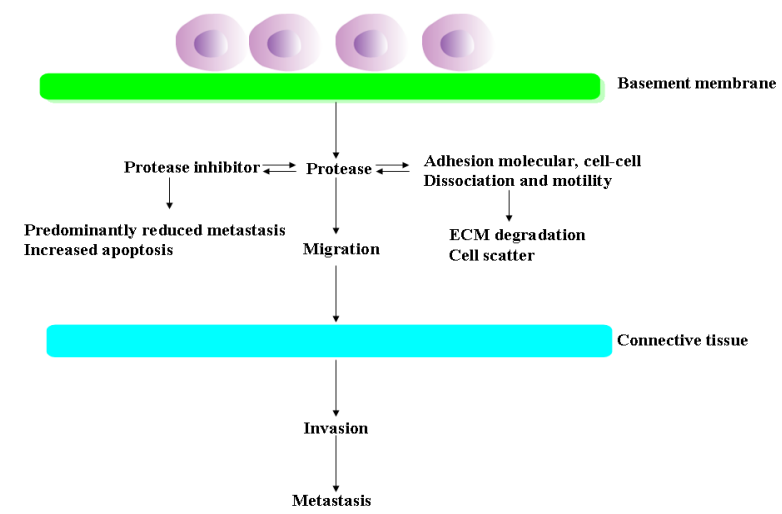

Figure 1. Schematic Representation of the Cells, Regulators, Adhesion Molecular to Protease Mediated Cancer Invasion and Metastasis level, such as microRNA-146b, microRNA-29b and microRNA-10b (Fang et al., 2011). The methylation status of several MMP genes suppressed MMPs expression (Chernov and Strongin, 2011). The proteolytic activity and the expression of most MMPs are normally low in healthy tissues. However, they are induced when ECM remodelling is required or injury consequently (Gialeli et al., 2011). Many studies have verified that most MMPs are secreted as inactive zymogens and shown to be activated by nitric oxide, cellular endopeptidases, reactive oxgen and phosphorylation (Gaffney et al., 2015). Moreover, the zymogen activity of MMPs is prevented by TIMPs.

Due to the ability of MMPs to degrade ECM and promote the migration of tumor endothelial from original sites, they were therefore considered to be an effective target for cancer treatment. However, the attempts to block MMPs directly and prevent tumor progression have failed in the extensive phase III clinical trials (Gialeli et al., 2011). Almost all MMP inhibitors (MMPIs) from multiple companies could not show clinical efficiency. Even worse, some compounds had severe side effects, such as inflammation, musculoskeletal pain, and joint strictures (Cao et al., 2011). In retrospect, the attempts to inhibit MMPs failed because they cleave not only ECM components but also many other proteins, including growth factor binding proteins, cytokine precursors, and chemokines. Therefore, it is not surprising that systemic and prolonged inhibition of MMP activities causes aberrant immune responses and other stromal reactions (Kitamura and Taketo, 2007). More recently, it has been recognized that the pathogenetic function of MMPs in cancer is far more complicated than initially conceived. Therefore, selection of specific proteases as targets for anticancer therapy needs to be performed carefully. Based on the lack of effectiveness of several broad spectrum MMPIs in advanced cancer types, these drugs have languished. Retrospective assessment of the design of these clinical trials has led to the recognition that specific MMPIs used in conjunction with cytotoxic chemotherapy in early stage rather than late stage cancer needs future consideration.

\section{Cysteine Cathepsin Proteases and its Small Molecular Inhibitors}

As mentioned above, people paid attention to the correlation between MMPs and cancer for years, overshadowed the potential contributions of other protease families in tumor. The papain family of cysteine cathepsin proteases has been recently validated as an important target in cancer (Monsouvanh et al., 2014). In addition, cysteine cathepsin inhibitors show potentially broader mechanistic efficacy and less toxicity as compared with the MMPIs (Gocheva and Joyce, 2007). The cathepsin family

\section{Table 1. Examples of Protease Species Commonly Implicated in Cancer Progression}

\begin{tabular}{lll}
\hline Class & Protease & Cancer indication \\
\hline Matrix metalloproteases & MMP-2,MMP-9,MMP-14 & Cancer progression; growth invasion and angiogenesis \\
Cysteine cathepsin proteases & Cathepsin B, Cathepsin L, Cathepsin S & Cancer invasion, growth and angiogenesis \\
& Cathepsin K & Tumor bone and breast cancer metastases \\
Plasmingen & PAI-1 & Invasion of tumor cells and tumor spreading \\
\hline
\end{tabular}


comprises 11 members: B, C, F, H, K, L, O, S, V, W and $\mathrm{X}$. They share a conserved active site that is formed by cysteine and histidine residues, and comprise three welldefined substrate binding sites (S2, S1 and S1') (Rothberg et al., 2013). Majority of cathepsins are endopeptidases, whereas some also exhibit exopeptidase activity (Jedeszko and Sloane, 2004). Most cathepsins have a proteindegrading function in the lysosomes in the majority of cell types. The activity of cathepsins is regulated by the balance between their endogenous inhibitors and activation of their inactive preproforms (Laurent-Matha et al., 2012). Like MMPs, specific cathepsins are often upregulated in cancer cells. Cathepsins can be expressed at the cell surface and secreted into the extracellular space, where they can degrade components of the ECM (Fonovic and Turk, 2014a). When attached to other cell surface proteins, cathepsins exert their proteolytic activity (Joyce et al., 2004). This extracellular activity allows cancer cells to invade surrounding tissues, blood and lymph vessels and metastasize to distant sites. Therefore, cathepsins are considered to be promising targets in anticancer therapy (Fricker, 2010). One possible mechanism by which cathepsins can be expressed at the cell membrane instead of intralysosomally, is via downregulation of molecular interactions with the insulin-like growth factor 2 receptor/ mannose-6-phosphate receptor (Coutinho et al., 2012). These receptors are responsible for transporting proteins into lysosomes. When expressions of these receptors are downregulated in cells, cathepsins can be shunted into the secretory pathway as occurs frequently in malignant cells.

Promising targets for anticancer therapy seem to be cathepsins B, C, H, K, L, S and X, because the levels of these cathepsins are elevated in cancer cells (Fonovic and Turk, 2014b). Cathepsins H and L are overexpressed in cancer cells only, in contrast to cathepsins B, C, S and X, which are also found to be overexpressed in non-malignant cells in tumors (Joyce et al., 2004; Strojnik et al., 2005). Cathepsins B and L have been studied most thoroughly, yet their functions are still not well-defined (Mohamed and Sloane, 2006). Goulet et al. demonstrated that cathepsins L isoforms are present in the nucleus, where they accelerate the progression of the cell into the $S$ phase by proteolytically processing the $\mathrm{CDP} / \mathrm{Cux}$ transcription factor (Goulet et al., 2007). This study suggested that cathepsin L could be a target in enhancing the effectiveness of chemotherapy.

The cysteine cathepsin inhibitors have been tested in animal models and clinical trials are nitriles, vinyl sulfones and epoxysuccinyl-based compounds (Elie et al., 2010). All these inhibitors are directed effects on the active site. According to their mechanism of action, the inhibitors can be further divided into the covalent or the non-covalent binders, and reversible or irreversible inhibitors. One of the first broad-spectrum cysteine cathepsin inhibitors to be studied was E-64-a covalent, irreversible, epoxysuccinyl-based inhibitor originally isolated from aspergillus japonicas (Wen et al., 2011). Limited experiment shave shown that E-64 can effectively inhibit cysteine cathepsins in vivo (Navab et al., 1997). In fact, the ethyl ester of E-64 (E-64d) was tested in clinical trials in Japanese individuals with muscular dystrophy, but
Potential Roles of Protease Inhibitors in Cancer Progression the trials were stopped at phase III of development owing to suboptimal performance (Fonovic and Turk, 2014b). GB111-NH2 is a novel cysteine cathepsin inhibitor, and serves as a therapeutic strategies for triggering tumorassociated macrophages cell death (Salpeter et al., 2015).

Further insights into pharmacological inhibitor design have come from the analysis of endogenous inhibitors of cysteine cathepsins: cystatins, stefins and kininogens (Turk et al., 2012). For example, a series of peptidyl-diazomethyl ketones has been designed on the basis of the structure of the segment of human cystatin $\mathrm{C}$ that interacts with the cysteine cathepsin active site (Wieczerzak et al., 2002). Moreover, Cathepsin S has recently received much attention as a tumor target due to its causative roles in the promotion of tumor invasion and upregulation in endothelial cells during angiogenesis (Gocheva et al., 2006). A cysteine protease cathepsin S specific antibody, Fsn0503h, has recently been shown to block ECM degradation and may regarded as a effective therapy of solid tumors (Vazquez et al., 2015).

The preclinical studies discussed here indicate that cysteine cathepsin inhibition will slow tumor growth, in part, by decreasing angiogenesis and invasion. To achieve substantial tumor regression, particularly in late-stage patients, it is likely that cysteine cathepsin inhibitors will need to be combined with additional therapies directed against the cancerous cells such as chemotherapy, radiation or biological agents (e.g. inhibitors of growth factor receptors) (Quail and Joyce, 2013). Furthermore, if cysteine cathepsin inhibitors predominantly target stromal cells in the tumor microenvironment, then evaluation of the patient response might be more accurately measured in terms of long-term disease stabilization, rather than short-term tumor shrinkage. The production of cysteine cathepsins and other proteases by infiltrating stromal cells, as opposed to cancer cells, could provide an additional therapeutic benefit since the coopted stromal cells should be genetically stable, and thus should not acquire drug resistance (Joyce, 2005).

\section{Plasminogen and Inhibitors}

The plasminogen system is composed of an inactive proenzyme plasminogen $(\mathrm{Plg})$ that can be converted to plasmin by either of two plasminogen activators (PA): urokinase-type (uPA) and tissue-type (tPA) plasminogen activators which are serine proteinases (Hildenbrand et al., 2009). Their activities are controlled by plasminogen activator inhibitors, PAI-1 and PAI-2 (plasminogen activator inhibitor-type 1 and type 2) belonging to the serine proteinase inhibitor (serpin) family. Plasmin displays a broad spectrum activity, and is able to degrade many glycoproteins and proteoglycans of ECM as well as fibrin, and to activate other proteases such as prometalloproteinases (Oh et al., 2002).

Plasminogen present at high concentration (1.5$2 \mathrm{mM}$ ) in plasma and interstitial fluids, is a plentiful potential source of plasmin activity. Cellular receptor for plasminogen has been identified and might localize plasmin proteolysis to the cell surface (Jones et al., 2004). Proteolytic activity driven by uPA is commonly 
recognized as an important factor in metastasis and angiogenesis (Kacsinta et al., 2014). The uPA system contains the following elements: (a) plasminogen, the enzyme in its nonactive form is called plasminogen and in the active form is called plasmin. Plasmin is a strong proteolytic enzyme, and it also activates other latent proteolytic enzymes, broadening the spectrum of proteins attacked. Plasmin is a key enzyme in the tumor invasion and the development of distant metastasis (Deryugina and Quigley, 2012); (b) activators, uPA and tPA. Both are weak proteolytic enzymes that activate plasminogen into plasmin by proteolytic cleavage. uPA is involved in pericellular proteolysis under a variety of physiological and pathological conditions. tPA mediates mainly intravascular thrombolysis (Yepes et al., 2009); (c) inhibitors of plasminogen activators, there are four known inhibitors of uPA: PAI-1, PAI-2, PAI-3, and a protein called nexin. Most relevant in the metastatic process is PAI-1 (Jankun et al., 2012); (d) the binding site of uPA is called the uPAR. High numbers of uPARs on the surface of cells, if occupied by uPA, create high proteolytic activity in the proximity of cancer cells, has been found in human cancer cell lines with metastatic behavior (Campodonico et al., 2010). The other plasminogen activator, tPA, does not seem to be relevant in the metastatic process (Murray et al., 2010).

PAI-1, a member of the serpin family of serine protease inhibitors, inhibits both tPA and uPA. From animal experiments as well as from in vitro data, the "pro-angiogenic" property of PAI-1 seems to be one of the prime candidates for the mechanism by which PAI1 causes "bad" clinical outcome and therefore a more "malignant" phenotype in several cancers (Hildenbrand et al., 2010). This mechanism would represent the response of the host to PAI-1 secreted by the tumor. With respect to PAI-1 effects on the tumor cell, most data point towards mechanism that involves ligation of PAI-1-uPA complexes to a member of the LDLR family, likely the VLDLR and LRP-1 (Jensen et al., 2006). Thereby a cryptic binding site in PAI-1 for the LDLR family member would be revealed by complex formation of PAI-1 with UPA and modulation of cellular signaling, initiated by uPAR, would occur. Signaling cascades thereby modulated include primarily the MAP kinase pathways as well as the JAK/ STAT pathway and lead to a change in cell adhesion and migration (Hsieh et al., 2010). Therefore, PAI-1 secreted by tumor cells would stimulate in a paracrine fashion angiogenesis guaranteeing sufficient supply of the growing tumor with oxygen and nutrients and in an autocrine fashion would modulate cellular signaling leading to increased cell adhesion and migration reflected by increased metastasis formation. From this model, interference with PAI-1 signaling would be a promising approach for an alternative, additional tumor therapy.

\section{Summary}

In view of the vast amount of literature concerning the role of MMPs, cathepsin, plasminogen and the relevant inhibition in cancer, we suggest that the main focus of future research in this matter should be aimed at the combination of protease inhibition and chemotherapy. More insights are needed into the effects of this strategy at a molecular, cellular and histological level. In addition, studies should be performed using different combinations, in order to find out what specific strategy is most promising for future clinical trials.

\section{Acknowledgements}

This study was supported by the National Natural Science Foundation of China (No. 31271516, No. 31201072), Shanxi Province Science Foundation for Youths (2012021028-4), Zhejiang Province Science Foundation (LY15H280008), The R\&D Infrastructure and Facility Development Program of Shanxi Province (2015091015), and Scientific and Technological Innovation Programs of Higher Education Institutions in Shanxi (2015175).

\section{References}

Alitalo A, Detmar M (2012). Interaction of tumor cells and lymphatic vessels in cancer progression. Oncogene, $\mathbf{3 1}$, 4499-508.

Behm B, Babilas $P$, Landthaler M, et al (2012). Cytokines, chemokines and growth factors in wound healing. $J$ Eur Acad Dermatol Venereol, 26, 812-20.

Campodonico PB, de Kier Joffe ED, Urtreger AJ, et al (2010). The neural cell adhesion molecule is involved in the metastatic capacity in a murine model of lung cancer. $\mathrm{Mol}$ Carcinog, 49, 386-97.

Cao XL, Xu RJ, Zheng YY, et al (2011). Expression of type IV collagen, metalloproteinase-2, metalloproteinase-9 and tissue inhibitor of metalloproteinase-1 in laryngeal squamous cell carcinomas. Asian Pac J Cancer Prev, 12, 3245-9.

Chernov AV, Strongin AY (2011). Epigenetic regulation of matrix metalloproteinases and their collagen substrates in cancer. Biomol Concepts, 2, 135-47.

Coutinho MF, Prata MJ, Alves S (2012). A shortcut to the lysosome: the mannose-6-phosphate-independent pathway. Mol Genet Metab, 107, 257-66.

Deryugina EI, Quigley JP (2012). Cell surface remodeling by plasmin: a new function for an old enzyme. J Biomed Biotechnol, 2012, 564259.

Elie BT, Gocheva V, Shree T, et al (2010). Identification and preclinical testing of a reversible cathepsin protease inhibitor reveals anti-tumor efficacy in a pancreatic cancer model. Biochimie, 92, 1618-24.

Fang JH, Zhou HC, Zeng C, et al (2011). MicroRNA-29b suppresses tumor angiogenesis, invasion, and metastasis by regulating matrix metalloproteinase 2 expression. Hepatology, 54, 1729-40.

Fonovic M, Turk B (2014a). Cysteine cathepsins and extracellular matrix degradation. Biochim Biophys Acta, 1840, 2560-70.

Fonovic M, Turk B (2014b). Cysteine cathepsins and their potential in clinical therapy and biomarker discovery. Proteomics Clin Appl, 8, 416-26.

Fricker SP (2010). Cysteine proteases as targets for metal-based drugs. Metallomics, 2, 366-77.

Gaffney J, Solomonov I, Zehorai E, et al (2015). Multilevel regulation of matrix metalloproteinases in tissue homeostasis indicates their molecular specificity in vivo. Matrix Biol.

Gearing AJ, Beckett $P$, Christodoulou M, et al (1994). Processing of tumour necrosis factor-alpha precursor by metalloproteinases. Nature, 370, 555-7. 
Gialeli C, Theocharis AD, Karamanos NK (2011). Roles of matrix metalloproteinases in cancer progression and their pharmacological targeting. FEBS J, 278, 16-27.

Gocheva V, Joyce JA (2007). Cysteine cathepsins and the cutting edge of cancer invasion. Cell Cycle, 6, 60-4.

Gocheva V, Zeng W, Ke D, et al (2006). Distinct roles for cysteine cathepsin genes in multistage tumorigenesis. Genes Dev, 20, 543-56.

Goulet B, Sansregret L, Leduy L, et al (2007). Increased expression and activity of nuclear cathepsin $\mathrm{L}$ in cancer cells suggests a novel mechanism of cell transformation. Mol Cancer Res, 5, 899-907.

Gupta SC, Kim JH, Prasad S, et al (2010). Regulation of survival, proliferation, invasion, angiogenesis, and metastasis of tumor cells through modulation of inflammatory pathways by nutraceuticals. Cancer Metastasis Rev, 29, 405-34.

Hanahan D, Weinberg RA (2011). Hallmarks of cancer: the next generation. Cell, 144, 646-74.

Hart JR, Liao L, Yates JR, 3rd, et al (2011). Essential role of Stat 3 in PI3K-induced oncogenic transformation. Proc Natl Acad Sci U S A, 108, 13247-52.

Hildenbrand R, Allgayer H, Marx A, et al (2010). Modulators of the urokinase-type plasminogen activation system for cancer. Expert Opin Investig Drugs, 19, 641-52.

Hildenbrand R, Schaaf A, Dorn-Beineke A, et al (2009). Tumor stroma is the predominant uPA-, uPAR-, PAI-1-expressing tissue in human breast cancer: prognostic impact. Histol Histopathol, 24, 869-77.

Hsieh MJ, Chen KS, Chiou HL, et al (2010). Carbonic anhydrase XII promotes invasion and migration ability of MDAMB-231 breast cancer cells through the p38 MAPK signaling pathway. Eur J Cell Biol, 89, 598-606.

Jankun J, Al-Senaidy A, Skrzypczak-Jankun E (2012). Can inactivators of plasminogen activator inhibitor alleviate the burden of obesity and diabetes? (Review). Int J Mol Med, 29, 3-11.

Jedeszko C, Sloane BF (2004). Cysteine cathepsins in human cancer. Biol Chem, 385, 1017-27.

Jensen JK, Malmendal A, Schiott B, et al (2006). Inhibition of plasminogen activator inhibitor-1 binding to endocytosis receptors of the low-density-lipoprotein receptor family by a peptide isolated from a phage display library. Biochem $J$, 399, 387-96.

Jones AL, Hulett MD, Altin JG, et al (2004). Plasminogen is tethered with high affinity to the cell surface by the plasma protein, histidine-rich glycoprotein. J Biol Chem, 279 38267-76.

Joyce JA (2005). Therapeutic targeting of the tumor microenvironment. Cancer Cell, 7, 513-20.

Joyce JA, Baruch A, Chehade K, et al (2004). Cathepsin cysteine proteases are effectors of invasive growth and angiogenesis during multistage tumorigenesis. Cancer Cell, 5, 443-53.

Kacsinta AD, Rubenstein CS, Sroka IC, et al (2014). Intracellular modifiers of integrin alpha $6 p$ production in aggressive prostate and breast cancer cell lines. Biochem Biophys Res Commun, 454, 335-40.

Kitamura T, Taketo MM (2007). Keeping out the bad guys: gateway to cellular target therapy. Cancer Res, 67, 10099102.

Laurent-Matha V, Huesgen PF, Masson O, et al (2012). Proteolysis of cystatin $\mathrm{C}$ by cathepsin $\mathrm{D}$ in the breast cancer microenvironment. Faseb J, 26, 5172-81.

Li Z, Zhang L, Zhao Y, et al.(2013). Cell surface GRP78 facilitates colorectal cancer cell migration and invasion. IN J Biochem Cell Biol, 45, 987-94.

Lu $P$, Weaver VM, Werb Z (2012). The extracellular matrix: a dynamic niche in cancer progression. J Cell Biol, 196 ,
DOI:http://dx.doi.org/10.7314/APJCP.2015.16.18.8047

Potential Roles of Protease Inhibitors in Cancer Progression 395-406.

Mohamed MM, Sloane BF (2006). Cysteine cathepsins: multifunctional enzymes in cancer. Nat Rev Cancer, 6 , 764-75.

Monsouvanh A, Proungvitaya T, Limpaiboon T, et al (2014). Serum cathepsin B to cystatin $\mathrm{C}$ ratio as a potential marker for the diagnosis of cholangiocarcinoma. Asian Pac J Cancer Prev, 15, 9511-5.

Murray IA, Krishnegowda G, DiNatale BC, et al (2010). Development of a selective modulator of aryl hydrocarbon (Ah) receptor activity that exhibits anti-inflammatory properties. Chem Res Toxicol, 23, 955-66.

Navab R, Mort JS, Brodt $P$ (1997). Inhibition of carcinoma cell invasion and liver metastases formation by the cysteine proteinase inhibitor E-64. Clin Exp Metastasis, 15, 121-9.

Nissinen L, Kahari VM (2014). Matrix metalloproteinases in inflammation. Biochim Biophys Acta, 1840, 2571-80.

Oh CK, Ariue B, Alban RF, et al (2002). PAI-1 promotes extracellular matrix deposition in the airways of a murine asthma model. Biochem Biophys Res Commun, 294, 115560.

Quail DF, Joyce JA (2013). Microenvironmental regulation of tumor progression and metastasis. Nat Med, 19, 1423-37.

Rakashanda S, Qazi AK, Majeed R, et al (2013). Antiproliferative activity of Lavatera cashmeriana- protease inhibitors towards human cancer cells. Asian Pac J Cancer Prev, 14, 3975-8.

Revach OY, Geiger B (2014). The interplay between the proteolytic, invasive, and adhesive domains of invadopodia and their roles in cancer invasion. Cell Adh Migr, 8, 215-25.

Rothberg JM, Bailey KM, Wojtkowiak JW, et al (2013). Acid-mediated tumor proteolysis: contribution of cysteine cathepsins. Neoplasia, 15, 1125-37.

Salpeter SJ, Pozniak Y, Merquiol E, et al (2015). A novel cysteine cathepsin inhibitor yields macrophage cell death and mammary tumor regression. Oncogene.

Sanman LE, Bogyo M (2014). Activity-based profiling of proteases. Annu Rev Biochem, 83, 249-73.

Shay G, Lynch CC, Fingleton B (2015). Moving targets: Emerging roles for MMPs in cancer progression and metastasis. Matrix Biol.

Shuman Moss LA, Jensen-Taubman S, Stetler-Stevenson WG (2012). Matrix metalloproteinases: changing roles in tumor progression and metastasis. Am J Pathol, 181, 1895-9.

Strojnik T, Kavalar R, Trinkaus M, et al (2005). Cathepsin L in glioma progression: comparison with cathepsin B. Cancer Detect Prev, 29, 448-55.

Turk V, Stoka V, Vasiljeva O, et al (2012). Cysteine cathepsins: from structure, function and regulation to new frontiers. Biochim Biophys Acta, 1824, 68-88.

van Horssen R, Buccione R, Willemse M, et al (2013). Cancer cell metabolism regulates extracellular matrix degradation by invadopodia. Eur J Cell Biol, 92, 113-21.

Vazquez R, Astorgues-Xerri L, Bekradda M, et al (2015). Fsn0503h antibody-mediated blockade of cathepsin S as a potential therapeutic strategy for the treatment of solid tumors. Biochimie, 108, 101-7.

Wen J, Nikitakis NG, Chaisuparat R, et al (2011). Secretory leukocyte protease inhibitor (SLPI) expression and tumor invasion in oral squamous cell carcinoma. Am J Pathol, 178, 2866-78.

Wieczerzak E, Drabik $P$, Lankiewicz L, et al (2002). Azapeptides structurally based upon inhibitory sites of cystatins as potent and selective inhibitors of cysteine proteases. J Med Chem, 45, 4202-11.

Yadav L,Puri N, Rastogi V, et al (2014). Matrix metalloproteinases and cancer - roles in threat and therapy. Asian Pac J Cancer Prev, 15, 1085-91. 
Peng Yang et al

Yan C, Boyd DD (2007). Regulation of matrix metalloproteinase gene expression. J Cell Physiol, 211, 19-26.

Yang $P$, Li Z, Fu R, et al (2014). Pyruvate kinase M2 facilitates colon cancer cell migration via the modulation of STAT3 signalling. Cell Signal, 26, 1853-62.

Yepes M, Roussel BD, Ali C, et al (2009). Tissue-type plasminogen activator in the ischemic brain: more than a thrombolytic. Trends Neurosci, 32, 48-55. 